\title{
Prevalence of Chronic Kidney Disease, Thrombotic Cardiovascular Events, and Use of Oral P2Y 12 Inhibitors among Veterans
}

\author{
Manisha Singh ${ }^{a, b}$ Deepa Raghavan ${ }^{a, b}$ James S. Williams ${ }^{b}$ Bradley C. Martin ${ }^{a}$ \\ Teresa J. Hudson ${ }^{\mathrm{a}}$ b Richard R. Owen ${ }^{\mathrm{b}}$ Nishank Jain ${ }^{\mathrm{a}, \mathrm{b}}$ \\ a University of Arkansas for Medical Sciences, Little Rock, AR, USA; ${ }^{b}$ Central Arkansas Veterans Affairs Healthcare \\ System, Little Rock, AR, USA
}

\section{Keywords}

Cardiovascular · Kidney · Outcomes · $\mathrm{P}_{2} \mathrm{Y}_{12}$ inhibitors ·

Prevalence

\begin{abstract}
Background: Contemporary prevalence of chronic kidney disease (CKD) and thrombotic cardiovascular (CV) events remains unclear in Veterans enrolled in the Veterans Affairs Health Care System (VA) care. Although oral P2 $\mathrm{Y}_{12}$ inhibitors (P2Y12i) are increasingly being prescribed to this patient population, the overall prescription trend for $\mathrm{P} 2 \mathrm{Y} 12 \mathrm{i}$ remains unclear. Methods: Using national VA corporate warehouse data, we used International Classification of Diseases-9 codes to identify Veterans with CKD, dialysis-dependent CKD, and CV events. VA pharmacy data were used to count P2Y12i prescriptions for the federal fiscal years (FY) 2011 through 2015. Results: The period prevalence of Veterans with CKD was 378,233 (6.1\%). The point prevalence of CKD increased by $49 \%$ from 132,979 (2.30\%) in FY11 to 213,444 (3.42\%) in FY15. The period prevalence of Veterans with dialysis-dependent CKD was 150,298 (2.4\%). In all, 128,703 (56.7\%) CV events occurred in Veterans with CKD. Veterans with CKD were given $50.1 \%$ of prescriptions for clopidogrel, $49.3 \%$ for prasugrel, and $60.4 \%$ for ticagrelor. In this patient
\end{abstract}

population, year-to-year increases in $\mathrm{P} 2 \mathrm{Y} 12 \mathrm{i}$ prescriptions were observed with a dramatic increase in ticagrelor prescriptions. Conclusion: CKD is common among Veterans and its true prevalence is likely being underestimated. The prevalence of dialysis-dependent CKD is higher among Veterans than the non-Veteran US population. CV events are widely co-prevalent and these patients are commonly prescribed P2Y12i. The recent increase in ticagrelor prescriptions in this patient population and large cost differences between the 3 P2Y12i underline the need for future studies to identify the preferred P2Y12i for these patients.

(C) 2018 The Author(s)

Published by S. Karger AG, Basel

\section{Background}

Chronic kidney disease (CKD) is one of the fastest growing chronic diseases and is the ninth leading cause of death in the United States [1]. Patients with CKD are at disproportionately higher risk of thrombotic cardiovascular $(\mathrm{CV})$ events than the general population and are more likely to die from such events than survive long enough to progress to dialysis-dependence [2]. The Veteran population is a special sub-group with commonly coexisting coronary heart disease and CKD

\begin{tabular}{|c|c|}
\hline KARGER & $\begin{array}{l}\text { (c) } 2018 \text { The Author(s) } \\
\text { Published by S. Karger AG, Basel }\end{array}$ \\
\hline $\begin{array}{l}\text { E-Mail karger@karger.com } \\
\text { www.karger.com/ajn }\end{array}$ & $\begin{array}{l}\text { This article is licensed under the Creative Commons Attribution- } \\
\text { NonCommercial-NoDerivatives } 4.0 \text { International License (CC BY- } \\
\text { NC-ND) (http://www.karger.com/Services/OpenAccessLicense). } \\
\text { Usage and distribution for commercial purposes as well as any dis- } \\
\text { tribution of modified material requires written permission. }\end{array}$ \\
\hline
\end{tabular}

Nishank Jain, MD, MPH

University of Arkansas for Medical Sciences

4301 W Markham St., Slot 501

Little Rock, AR 72205 (USA)

E-Mail NJain2@uams.edu 
probably related to their old age and high burden of comorbidities such as diabetes and hypertension. Between April 2002 and September 2006, 5,760 Veterans in the Veterans Health Administration (VA) received percutaneous coronary interventions (PCIs) per year [3]; this number doubled to 10,715 per year in the period from October 2007 to September 2013 [4]. Thrombotic CV events in CKD patients portend poor prognosis such that at 1 -year post-PCI, Veterans with CKD had a $75 \%$ higher risk of death or thrombotic $\mathrm{CV}$ event compared to Veterans with normal kidney function, adjusted hazard ratio of 1.75 (95\% CI 1.51-2.04) [3]. Veterans with CKD experience increased rates of hospitalization, adverse clinical outcomes, high disease burden to healthcare services, and high health care-related costs [5]. However, the prevalence of CKD and its co-prevalence with thrombotic CV events among Veterans remain unknown.

Management of thrombotic CV events frequently involves PCIs and prescriptions for aspirin with an oral $\mathrm{P}_{2} \mathrm{Y}_{12}$ inhibitor (P2Y12i). The recent update of the American Heart Association Guidelines for acute coronary syndrome recommends the use of ticagrelor rather than other P2Y12i compounds including clopidogrel for maintenance antiplatelet therapy in patients treated with PCIs or medical therapy alone [6]. However, the VA Pharmacy Benefits Management Service guidelines continue to recommend the use of clopidogrel in Veterans with acute coronary syndrome and it is the only formulary P2Y12i within the VA system [7]. Although there are large cost differences between the P2Y12i drugs, the overall prescription trends for these drugs among Veterans with CKD remain unknown.

In 2015, the VA proposed a plan to the US Congress for transitioning into a high-performance network where high-value health care organization (VA) and providers serve high-value care to a defined Veteran population [8]. To accomplish such goals, it is important to estimate disease burden for some of the rapidly growing chronic diseases such as CKD. In addition, it is also important to identify prescription trends of P2Y12i in Veterans with CKD especially when this sub-group of Veteran population is most likely to receive these prescriptions, given the large price differences between P2Y12i and the unclear benefits associated with their use. Our objectives were to determine 3 interrelated aspects of CVD among Veterans with CKD: (1) period prevalence of CKD among Veterans enrolled in VA care during the study period, (2) coprevalence of CV events and CKD, and (3) overall prescription trends of P2Y12i in Veterans with CKD.

\section{Methods and Results}

After Institutional Review Board approval from the Central Arkansas Veterans Affairs Health Care System, we requested for VA Corporate Data Warehouse (CDW) data. Ticagrelor first became available in the market in federal fiscal year (FY) 2011, which was chosen as the beginning of the study period. The study end date was chosen when the switch from the International Code of Diseases (ICD)-9 to ICD-10 codes occurred at the end of FY15. We used the unique Veteran identification codes to determine the number of Veterans enrolled in VA care per FY. Any appearance of validated ICD-9 codes 585.x and V45.11 linked to unique Veteran identification codes in the CDW data during the study period allowed us to identify Veterans with CKD. The "CKD" cohort included both, non-dialysis CKD and dialysis-dependent CKD. Then, we searched for thrombotic CV and cerebrovascular events using validated ICD-9 codes in the cohort: acute myocardial infarction (410.xx), unstable angina or intermediate coronary syndrome (411.1x), ischemic heart disease (414.x), and occlusion and stenosis of cerebral arteries (433-5). Finally, we used VA pharmacy drug codes and generic names of drugs to identify prescriptions for aspirin and P2Y12i (clopidogrel, prasugrel and ticagrelor) in the cohort. We calculated the percent annual increase in the number of prescriptions for P2Y12i as the difference in the prescription counts between consecutive FY.

The overall period prevalence of unique Veterans with CKD code was 378,233 (6.1\%) during FY11-15. The point prevalence of CKD among total Veteran population enrolled in VA care increased by $49 \%$ from 132,979 (2.30\%) in FY11 to 213,444 (3.42\%) in FY15 (Fig. 1). The overall period prevalence of Veterans with dialysis-dependent CKD was 150,298 (2.4\%), which is much higher than the general US population $(<1 \%)$ [9]. There was slight decrease in the point prevalence of Veterans with dialysis-dependent CKD from 29,554 (0.51\%) in FY11 to 30,670 (0.49\%) in FY15 (Fig. 1). During FY11-15, there were 226,982 CV events recorded among Veterans enrolled in VA care. Of these, 128,703 (56.7\%) occurred in Veterans with concurrent diagnosis of CKD.

Of the 378,233 Veterans with CKD in FY11-15, 66.7\% were on aspirin and 25\% received prescriptions for P2Y12i-97\% for clopidogrel, $1 \%$ for ticagrelor, and 2\% for prasugrel. During FY11-15, $50.1 \%$ of prescriptions for clopidogrel, $49.3 \%$ for prasugrel, and $60.4 \%$ for ticagrelor were associated with CKD diagnosis (Table 1). We observed year-to-year increases in the prescriptions for P2Y12i with a dramatic increase in prescriptions for ticagrelor among Veterans with CKD (Fig. 2).

\section{Comments}

We report a $49 \%$ increase in the point prevalence of CKD among Veterans from $2.30 \%$ in FY11 to $3.42 \%$ in FY15. Exclusive use of ICD-9 codes instead of laboratory measures of serum creatinine and albuminuria to identify CKD may grossly underestimate the presence of CKD. That would explain why the point prevalence in our study is far lower than $14.8 \%$ reported in US Renal Data System, which employs diagnostic methods to identify
68

Am J Nephrol 2018;47:67-71 DOI: $10.1159 / 000486647$
Singh/Raghavan/Williams/Martin/ Hudson/Owen/Jain 
Fig. 1. Line graph showing the number (yaxis) of the total Veteran population enrolled in VA care (circle), Veterans with chronic kidney disease (square) and Veterans with dialysis-dependent chronic kidney disease (triangle) during fiscal years 2011 through 2015 (x-axis). Percentages on the blue and black line show proportion of Veterans with chronic kidney disease and dialysis-dependent chronic kidney disease, respectively. Please see online version for color image.

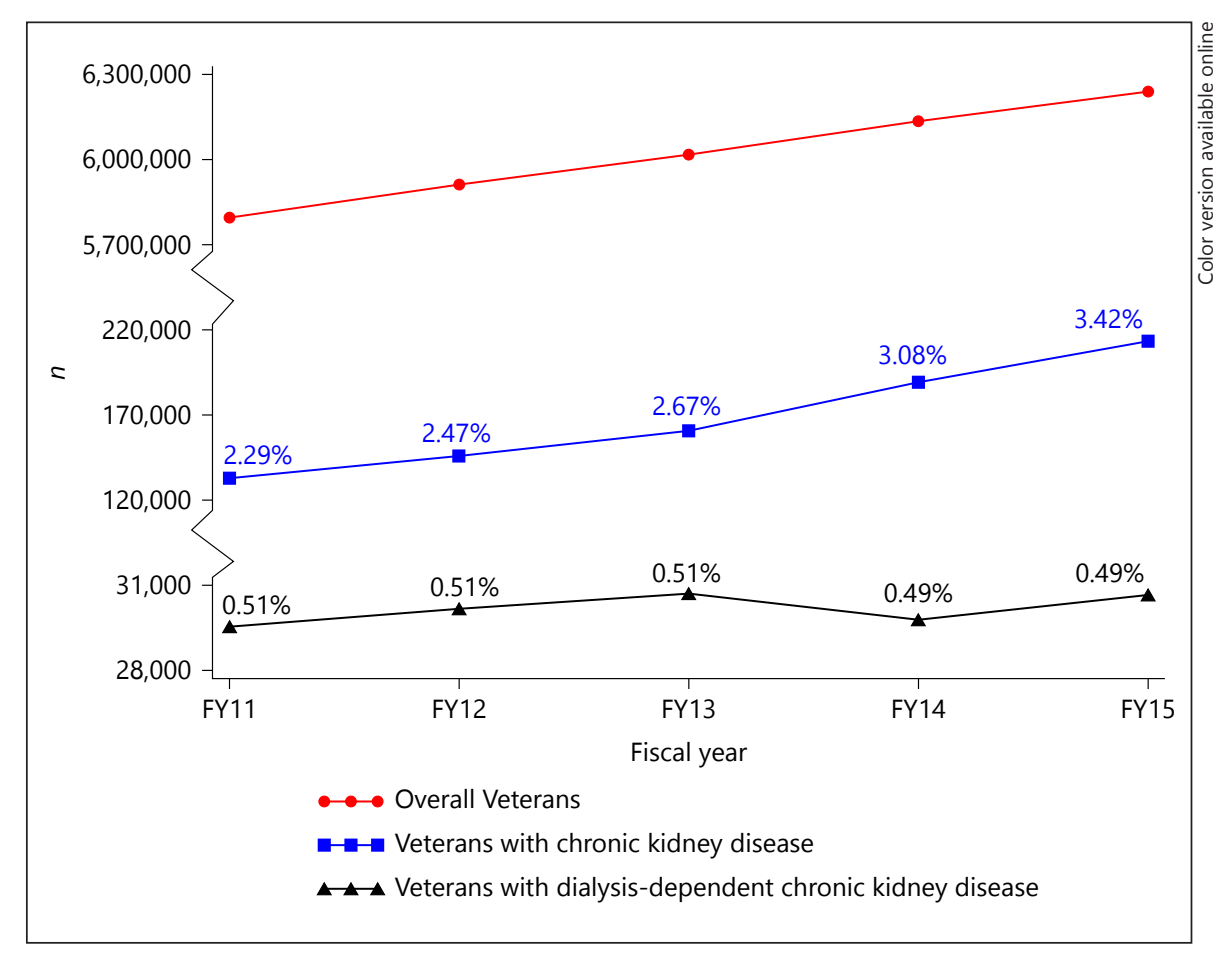

CKD including serum creatinine-based glomerular filtration rate formula and urine albumin-to-creatinine ratios [9]. One of the reasons for the increase in period prevalence of CKD among Veterans could be change in physician behavior and more frequent use of CKD diagnoses codes in VA medical records. This would suggest that $\mathrm{CKD}$ is underdiagnosed among Veterans or be a reflection of inaccurate documentation/coding in the study population. On the other hand, we found the period prevalence of dialysis-dependent CKD to be much higher than the general population $(2.4$ vs. $<1 \%)$. Our findings are similar to recent studies that reported higher incidence of dialysis-dependent CKD among Veterans compared to the non-Veteran population $[10,11]$. A majority of the Veteran population that develops dialysis-dependent CKD chooses VA as the primary payer over Medicare, given the large differences in out-of-pocket medication co-payments or reimbursements, which is much less in the VA. Therefore, we believe that our data is more likely to be a true estimate of the prevalence of dialysisdependent CKD among Veterans enrolled in VA care. Aging, higher comorbidity index, or Veteran-specific risk factors such as higher prevalence of mental illness, substance abuse, lower educational status, or lower socioeconomic status may explain the higher prevalence rate of dialysis-dependent CKD among Veterans compared to
Table 1. Total number of prescriptions for $\mathrm{P} 2 \mathrm{Y}_{12}$ inhibitors (P2Y12i) administered through the Veterans Health Administration (VA) pharmacies during federal fiscal years 2011-2015

\begin{tabular}{lll}
\hline P2Y12i & $\begin{array}{l}\text { Total number of prescriptions administered } \\
\text { through the VA pharmacies }\end{array}$ \\
\cline { 2 - 3 } & $\begin{array}{l}\text { Veterans enrolled } \\
\text { in VA care, } \mathrm{n}\end{array}$ & $\begin{array}{l}\text { Sub-group of Veterans } \\
\text { with associated CKD } \\
\text { diagnosis code, } \%\end{array}$ \\
\hline $\begin{array}{ll}\text { Clopidogrel } \\
\text { Prasugrel }\end{array}$ & 201,321 & 50.1 \\
Ticagrelor & 1,217 & 49.3 \\
\hline
\end{tabular}

the general population. However, these potential risk factors remain to be investigated as risk factors for the higher prevalence of dialysis-dependent CKD among Veteran population.

Based on the USRDS 2016 annual report, the prevalence of coronary heart disease is twofold higher among CKD population compared to the general population [9]. Hence, it is not surprising that we found $56.7 \%$ of thrombotic CV events to be associated with CKD diagnosis. This may be an underestimation of the true burden of thrombotic CV events among Veterans with CKD as only 
Fig. 2. Line graph showing total number of prescriptions (y-axis) for clopidogrel (circles), prasugrel (squares) and ticagrelor (triangles) administered to Veterans with chronic kidney disease during fiscal years 2011 through 2015 (x-axis). Percentages in the line graph indicate percent annual increases in the number of prescriptions for an oral $\mathrm{P}_{2} \mathrm{Y}_{12}$ inhibitor calculated as the difference in the prescription counts between consecutive fiscal years. Please see online version for color image.

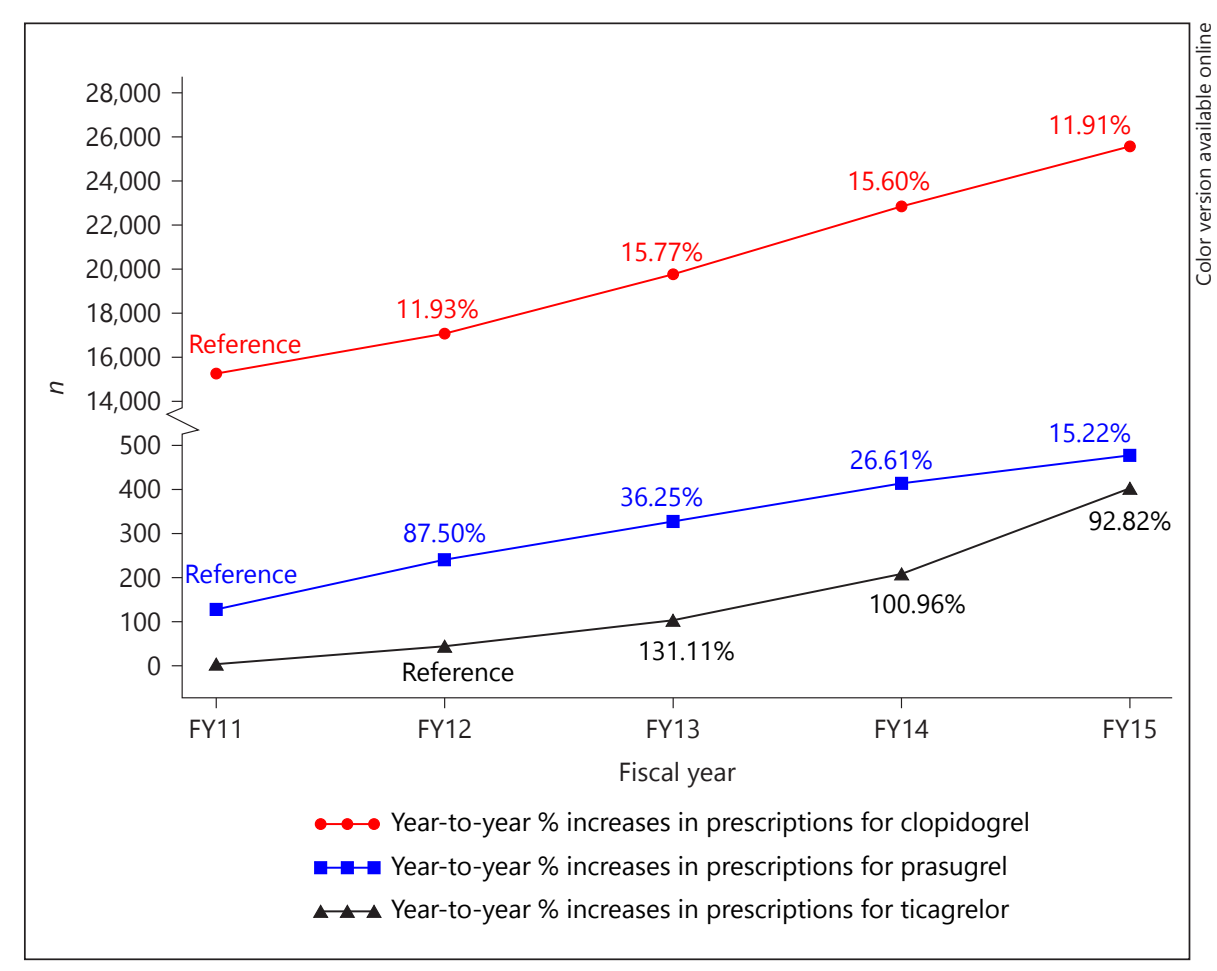

about half of the Veterans with CKD enrolled in VA care use either the VA system exclusively or dually with nonVA care [10]. Our data underline the importance of future CV research among Veterans with CKD - a subgroup that has been relatively understudied than the nonVeteran population.

We report that two thirds of Veterans with CKD were prescribed aspirin. Veterans may choose to purchase aspirin from another pharmacy over the VA pharmacy, as it may be cheaper outside the VA, and thus may not be captured accurately by the CDW. The number of prescriptions for aspirin may be an underestimate of the true prevalence of aspirin users among Veterans with CKD.

A recent sub-group analysis of the participants in the Platelet Inhibition and Patient Outcomes trial with creatinine clearance of $<60 \mathrm{~mL} / \mathrm{min}$ reported ticagrelor to be effective in reducing the composite outcome of $\mathrm{CV}$ death, acute myocardial infarction, or stroke at 12 -months by $23 \%$ over clopidogrel, adjusted hazard ratio 0.77 (95\% CI 0.65-0.90) [12]. Major bleeding episodes and fatal bleedings were not different among CKD participants treated with ticagrelor or clopidogrel. These findings might explain why we observed year-to-year increases in the prescriptions for P2Y12i with the largest and most dramatic increase in prescriptions for ticagrelor among Veterans with CKD. Despite this guideline, the VA non-formulary prescriptions for prasugrel and ticagrelor are increasingly being used in Veterans with CKD. Interestingly, more than half of the total prescriptions for ticagrelor were prescribed to Veterans with CKD. Although, comparative effectiveness and safety data of P2Y12i were recently published in the general population reporting the superiority of ticagrelor over other P2Y12i, data remain limited among individuals with $\mathrm{CKD}$, especially dialysis-dependent or pre-dialysis CKD with creatinine clearance $<30$ $\mathrm{mL} / \mathrm{min}$, to support these prescribing trends observed in the VA system [13].

Strengths of our findings are that we used the national VA dataset from CDW to report our findings. Previous studies were limited to regional VA networks and only reported incidence of dialysis-dependent CKD. In addition, we used validated ICD-9 and drug codes to identify CKD, thrombotic CV events, and P2Y12i prescriptions. However, our findings also have some limitations. First, our findings do not include Veterans who were not enrolled in VA care or those enrolled in non-VA care exclusively. Second, using ICD-9 code to identify dialysis-dependent CKD may be fraught with limitations, as it does not confirm receipt of chronic outpatient dialysis treatment procedures. Third, we did not use serum laboratory data to confirm a clinical diagnosis of CKD. However, using ICD-9 codes to determine prevalence of chronic dis- 
eases is a validated method to report the prevalence of chronic diseases. Our goal was to briefly describe the overall prevalence of CKD and thrombotic CV events among Veterans enrolled in VA care and report overall trends of P2Y12i prescriptions among Veterans with CKD. Therefore, we did not collect baseline characteristics of individual Veterans for this epidemiological data report.

In conclusion, $\mathrm{CKD}$ is common among Veterans in the United States and its true prevalence in this cohort is likely being underestimated. The prevalence of dialysisdependent CKD is higher among Veterans than the general US population. Thrombotic CV events are widely coprevalent in Veterans with CKD and these patients are commonly prescribed P2Y12i. The recent increase in ti- cagrelor prescriptions among Veterans with CKD underlines the need for future studies, which specifically focus on identifying the most favorable P2Y12i in this high-risk population.

\section{Disclosure Statement}

The authors have no conflicts of interest to disclose.

\section{Grant Support}

This study was supported by the American Heart Association Scientist Development Grant 16SDG31000045 (N.J.).

\section{References}

1 Centers for Disease Control and Prevention (CDC): Kidney disease mortality-Michigan, 1989-2005. MMWR Morb Mortal Wkly Rep 2007;56:225-227.

2 Benjamin EJ, Blaha MJ, Chiuve SE, et al: Heart disease and stroke statistics-2017 update: a report from the American Heart Association. Circulation 2017;135:e146-e603.

3 Siddiqi OK, Smoot KJ, Dufour AB, et al: Outcomes with prolonged clopidogrel therapy after coronary stenting in patients with chronic kidney disease. Heart 2015;101:1569-1576.

4 Barnes GD, Stanislawski MA, Liu W, et al: Use of contraindicated antiplatelet medications in the setting of percutaneous coronary intervention: insights from the veterans affairs clinical assessment, reporting, and tracking program. Circ Cardiovasc Qual Outcomes 2016;9:406-413.

5 Jain N, Kotla S, Little BB, et al: Predictors of hyperkalemia and death in patients with cardiac and renal disease. Am J Cardiol 2012;109: 1510-1513.

6 Levine GN, Bates ER, Bittl JA, et al: 2016 ACC/ AHA Guideline Focused Update on Duration of Dual Antiplatelet Therapy in Patients With Coronary Artery Disease: A Report of the
American College of Cardiology/American Heart Association Task Force on Clinical Practice Guidelines: An update of the 2011 ACCF/AHA/SCAI guideline for percutaneous coronary intervention, 2011 ACCF/AHA guideline for coronary artery bypass graft surgery, 2012 ACC/AHA/ACP/AATS/PCNA/ SCAI/STS guideline for the diagnosis and management of patients with stable ischemic heart disease, 2013 ACCF/AHA guideline for the management of ST-elevation myocardial infarction, 2014 AHA/ACC guideline for the management of patients with non-ST-elevation acute coronary syndromes, and 2014 ACC/AHA guideline on perioperative cardiovascular evaluation and management of patients undergoing noncardiac surgery. Circulation 2016;134:e123-e155.

7 Clinical Guidance-Criteria for Ticagrelor Use: VA Pharmacy Benefit Management Services. https://www.pbm.va.gov/clinicalguidance/criteriaforuse.asp (accessed July 24, 2017 at 1:30 pm).

8 Califf RM, Robb MA, Bindman AB, et al: Transforming evidence generation to support health and health care decisions. N Engl J Med 2016;375:2395-2400.
9 US Renal Data System: 2016 Annual Data Report. Bethesda, National Institutes of Health, National Institute of Diabetes and Digestive and Kidney Diseases, 2017 (accessed July 24, 2017 at 1:20 pm)

10 Wang V, Maciejewski ML, Patel UD, Stechuchak KM, Hynes DM, Weinberger M: Comparison of outcomes for veterans receiving dialysis care from VA and non-VA providers. BMC Health Serv Res 2013;13:26.

11 Kalantar-Zadeh K, Crowley ST, Beddhu S, et al: Renal replacement therapy and incremental hemodialysis for veterans with advanced chronic kidney disease. Semin Dial 2017;30: 251-261.

12 James S, Budaj A, Aylward P, et al: Ticagrelor versus clopidogrel in acute coronary syndromes in relation to renal function: results from the Platelet Inhibition and Patient Outcomes (PLATO) trial. Circulation 2010;122: 1056-1067.

13 Gosling R, Yazdani M, Parviz Y, et al: Comparison of P2Y12 inhibitors for mortality and stent thrombosis in patients with acute coronary syndromes: single center study of 10793 consecutive 'real-world' patients. Platelets 2017;28:767-773. 